\title{
Digital terrain modeling with orthogonal polynomials*
}

\author{
I. V. Florinsky and A. N. Pankratov \\ iflor@mail.ru, pan@impb.ru \\ Institute of Mathematical Problems of Biology, Russian Academy of Sciences, \\ 1 Vitkevicha st., Pushchino, Moscow Region, Russia
}

\begin{abstract}
Background: Mathematical problems of digital terrain analysis include interpolation of digital elevation models (DEMs), DEM generalization and denoising, as well as computation of morphometric variables by calculation of partial derivatives of elevation. Traditionally, these procedures are based on numerical treatments of DEMs, two-dimensional (2D) discrete functions of elevation.

Methods: We developed a spectral analytical method and algorithm based on high-order orthogonal expansions using the Chebyshev polynomials of the first kind with the subsequent Fejér summation. The method and algorithm are intended for analytical treatment of regularly spaced DEMs, such as DEM global approximation, generalization, and denoising as well as computation of morphometric variables by analytical calculation of partial derivatives.

Results: To test the method and algorithm, a DEM of the Northern Andes containing 230,880 points (the elevation matrix $480 \times 481$ ) has been used. The DEMs were reconstructed with 480 , 240, 120, 60, and 30 expansion coefficients. The first and the second partial derivatives of elevation were analytically calculated from the reconstructed DEMs. The models of 14 local morphometric variables were then computed with the derivatives. A set of maps of elevation and horizontal curvature $\left(k_{h}\right)$ related to different number of expansion coefficients well illustrates data generalization effects, denoising, and removal of artifacts contained in the original DEM. Concluding Remarks: The test results demonstrated a good performance of the developed method and algorithm. They can be utilized as a universal tool for analytical treatment in digital terrain modeling.
\end{abstract}

Keywords: Chebyshev polynomials; Fejér summation; generalization; denoising; partial derivatives

DOI: $10.21469 / 22233792.1 .12 .01$

\section{Цифровое моделирование рельефа с использованием ортогональных полиномов}

\author{
И. В. Флоринский, А.Н. Панкратов \\ Институт математических проблем биологии РАН
}

\begin{abstract}
Математические аспекты цифрового моделирования рельефа включают интерполяцию цифровых моделей высот (ЦМВ), генерализацию и подавление шума в ЦМВ, а также расчет морфометрических характеристик на основе вычисления частных производных высоты. Эти процедуры традиционно основаны на численной обработке ЦМВ - дискретных функций двух переменных. Авторы разработали спектрально-аналитический метод и алгоритм на основе ортогональных разложений высокого порядка с использованием многочленов Чебышёва I рода с последующим суммированием Фейера. Метод и алгоритм предназначены для аналитической обработки ЦМВ, включая глобальную аппроксимацию
\end{abstract}

\footnotetext{
*The study was supported by RFBR grant 15-07-02484.
} 
регулярных ЦМВ, генерализацию и подавление шума в ЦМВ, а также расчет морфометрических характеристик на основе аналитического вычисления частных производных. Для тестирования метода и алгоритма использовалась ЦМВ Северных Анд, включающая 230880 точек (матрица высот $480 \times 481$ ). Цифровые модели высот были восстановлены с использованием 480, 240, 120, 60 и 30 коэффициентов разложения. Первые и вторые частные производные высоты были аналитически рассчитаны по восстановленным ЦМВ. Производные были использованы для расчета моделей горизонтальной кривизны $\left(k_{h}\right)$. Серия карт высоты и $k_{h}$, полученных для различного числа коэффициентов разложения, хорошо иллюстрирует генерализацию данных, подавление шума и удаление артефактов, присутствующих в исходной ЦМВ. Результаты тестирования показали высокую работоспособность предлагаемого метода и алгоритма. Они могут применяться как универсальное средство для аналитической обработки данных в цифровом моделировании рельефа.

Ключевые слова: полиномы Чебышёва; суммирование Фейера; генерализачия; подавление иума; частные производные

DOI: $10.21469 / 22233792.1 .12 .01$

\section{Introduction}

Topography is one of the main factors controlling processes taking place in the near-surface layer of the planet. In particular, topography is one of the soil forming factors since it influences: (a) climatic and meteorological characteristics which control hydrological and thermal regimes of soils; (b) prerequisites for gravity-driven overland and intrasoil lateral transport of water and other substances; and (c) spatial distribution of vegetation cover. At the same time, being a result of the interaction of endogenous and exogenous processes of different scales, topography can reflect the geological structure of a terrain. In this connection, digital terrain analysis and digital terrain models (DTMs) are widely used to solve various multiscale problems of geomorphology, hydrology, remote sensing, soil science, geology, geophysics, geobotany, glaciology, oceanology, climatology, planetology, and other disciplines [1-4].

Mathematical issues of quantitative modeling and analysis of the topographic surface can be summarized in three main problems:

(1) interpolation of irregularly and regularly spaced digital elevation models (DEMs), twodimensional (2D) discrete functions of an elevation defining the topographic surface as a set of values measured at the grid nodes. This task is commonly carried out by various local interpolation techniques (e.g., piecewise splines) [5-7];

(2) DEM filtering to denoise, generalize, and decompose DEMs into components of different spatial scales [4]. These tasks are usually attacked by 2D discrete Fourier transform [8-10], 2D discrete wavelet transform [11-13], and 2D singular spectrum analysis [14];

(3) derivation of local morphometric variables from regularly spaced DEMs to analyze the topographic surface and relationships between topography and other natural and artificial components of geosystems [4]. The list of local morphometric variables includes: slope gradient, slope aspect, minimal curvature, maximal curvature, mean curvature, the Gaussian curvature, unsphericity curvature, horizontal curvature, vertical curvature, difference curvature, accumulation curvature, ring curvature, vertical excess curvature, and horizontal excess curvature. If the topographic surface is defined by a continuous, single-valued function

$$
z=f(x, y)
$$


where $z$ is the elevation and $x$ and $y$ are the Cartesian coordinates, local morphometric variables are the functions of partial derivatives of elevation:

$$
r=\frac{\partial^{2} z}{\partial x^{2}} ; t=\frac{\partial^{2} z}{\partial y^{2}} ; s=\frac{\partial^{2} z}{\partial x \partial y} ; p=\frac{\partial z}{\partial x} ; q=\frac{\partial z}{\partial y} .
$$

For example, horizontal curvature $\left(k_{h}\right)$, one of the most important morphometric attributes, is calculated by the following equation [15]:

$$
k_{h}=-\frac{q^{2} r-2 p q s+p^{2} t}{\left(p^{2}+q^{2}\right) \sqrt{1+p^{2}+q^{2}}} .
$$

Equations of other local morphometric variables can be found elsewhere [4]. To compute $r$, $t, s, p$, and $q(2)$ from DEMs based on plane square grids or spheroidal equal angular grids, one can apply methods based on approximation of partial derivatives by finite differences using the $3 \times 3$ or $5 \times 5$ moving windows [15-19].

However, it is obvious that the three mathematical problems of digital terrain analysis may be resolved within a framework of an analytical treatment of DEMs using global approximation with (orthogonal) polynomials. In the 1960-1980s, there were attempts to apply high-order polynomials for DEM global approximation [20-23]. These attempts have faced several limitations. First, such approaches have required considerable computer resources. Second, practical tasks have demanded to work with increasingly large DEMs containing tens of thousands to a few million points; existed methods and computers could not handle such data amounts. Third, the topographic surface has appeared too complex for wide application of global polynomial approximations. As a result, only low-order orthogonal polynomials have been utilized in trend-surface analysis, a technique to reveal trend and residual components of the topographic surface $[24,25]$. However, the current progress in the theory and practice of polynomial approximation [26-28] suggests that it is now possible to solve such problems.

In this paper we describe a spectral analytical method and algorithm based on high-order orthogonal expansions using the Chebyshev polynomials of the first kind with the subsequent Fejér summation. The method and algorithm are intended for the analytical treatment of regularly spaced DEM, such as DEM global approximation, denoising, and generalization as well as computation of morphometric variables by analytical calculation of partial derivatives.

\section{Method}

Let consider a function of two variables (1) defined in a rectangular domain. To approximate analytically this function, the $2 \mathrm{D}$ expansion by the orthogonal Chebyshev polynomials of the first kind

$$
z=\sum_{i=0}^{l-1} \sum_{j=0}^{l-1} d_{i j} T_{i}(x) T_{j}(y),
$$

where $T_{i}(x)$ and $T_{i}(y)$ are the Chebyshev polynomials orthogonal in the interval $[-1,1]$ and $d_{i j}$ are the expansion coefficients, has been used. It is assumed that the domain of the initial function is translated into the domain of the orthogonal polynomials by a linear transformation.

The method and algorithms for the function expansion by the Chebyshev polynomials are described in $[29,30]$. To calculate expansion coefficients, we use an operator, or matrix method introduced and studied in [30]. In this study, a 2D approximation of the function (1) is performed as a superposition of one-dimensional approximations by each variable. 
We use the following formula for the Chebyshev polynomials:

$$
B=\left\{T_{0}=\frac{1}{\sqrt{2}}, T_{i}=\cos (i \arccos x)\right\}
$$

where $i$ is the number of basis function, $i=1, \ldots, l-1$. The system of functions $T_{i}(x)$, $i=0, \ldots, l-1$, satisfies the orthogonality condition in the scalar product defined as follows:

$$
\left(T_{i}, T_{j}\right)=\int_{-1}^{1} T_{i}(x) T_{j}(x) \frac{1}{\sqrt{1-x^{2}}} d x= \begin{cases}\pi & \text { if } i=j \\ 0 & \text { if } i \neq j\end{cases}
$$

In the discrete form, the scalar product on a nonuniform grid of $k$ nodes

$$
t_{i}=\cos \left(\frac{\pi(i-1 / 2)}{k}\right)
$$

which are the zeros of the orthogonal polynomial $T_{k}(x), i=1, \ldots, k$, has the form:

$$
\left(T_{n}, T_{m}\right)=\frac{2}{k} \sum_{i=1}^{k} T_{n}\left(t_{i}\right) T_{m}\left(t_{i}\right)= \begin{cases}\pi & \text { if } n=m ; \\ 0 & \text { if } n \neq m .\end{cases}
$$

The expansion coefficients are calculated by the expressions:

$$
c_{i}=\frac{\left(f, T_{i}\right)}{\left(T_{i}, T_{i}\right)} .
$$

Approximations based on orthogonal polynomials always lead to oscillatory artifacts due to the Gibbs phenomenon [31]. To solve this problem, the original approximation was replaced with a smooth representation obtained as an arithmetic mean of all partial sums of an orthogonal series (the Fejér summation). According to the Fejér theorem [32], the initial mean-square approximation becomes the uniform one in this representation. The Fejér summation (or averaging) is the powerful method to suppress or eliminate oscillatory artifacts of the Gibbs phenomenon in data sets approximated with polynomials [31,33].

Transformation of expansion coefficients corresponding to the arithmetic mean of the partial sums of an orthogonal series has the form:

$$
\tilde{c}_{i}=\frac{l-i}{l} c_{i}
$$

where $\tilde{c}_{i}$ are the new weighting coefficients of an orthogonal series, $i=0, \ldots, l-1$.

After the approximation of the function $z(1)$ and the suppression of oscillatory artifacts, it is possible to calculate the partial derivatives $r, t, s, p$, and $q(2)$ in an analytical representation similar to the function $z$, that is, in the form of $2 \mathrm{D}$ orthogonal series. For the case of the Chebyshev polynomials, calculation of the expansion coefficients of a derivative from the expansion coefficients of the initial function is described in [29]. In general form for an arbitrary basis, a coefficient conversion scheme is presented in [28].

In the present case, these formulas take the following form:

$$
p_{l-1}=0 ; \quad p_{l-2}=2(l-2) c_{l-1} ; p_{j}=p_{j+2}+2 j c_{j+1} ; \quad p_{0}=\frac{p_{0}}{\sqrt{2}}
$$

where $p_{j}$ are the expansion coefficients of the derivative of an orthogonal series, $j=l-3, \ldots, 0$. 
It should also be noted that expansion coefficients of the derivative should be scaled in the case of a linear transformation of function domain:

$$
p_{j}=\frac{2}{T} p_{j}
$$

where $T$ is the length of a function interval, $j=0, \ldots, l-1$.

Finally, to derive local morphometric variables, all the calculated values of the derivatives are substituted into related equations, for instance, the $k_{h}$ equation (3).

\section{Algorithm}

Let the initial array is specified as a matrix $A$ with the dimension $m \times n$ representing the function values at the nodes of a square grid; $x_{1}, \ldots, x_{m}$ are the values of the grid nodes in the interval $[-1,1]$ along abscissa, $y_{1}, \ldots, y_{n}$ are the values of the grid nodes in the interval $[-1,1]$ along ordinate; and $t_{1}, \ldots, t_{k}$ are the Gaussian quadrature nodes.

The $k$ values determine the maximum degree of expansion $l$ in Eq. (4). Since $m \approx n$, so, the Gaussian grid is identical for abscissas and ordinates. For a more accurate calculation of the expansion coefficients, it is recommended to choose the $k$ value greater than $m$ and $n$. In this study, $k=8 \max (m, n)$.

Let introduce the following notations: $L_{x t}$ is the matrix of linear interpolation from the grid $x$ to the grid $t ; T_{t}$ is the matrix of the Chebyshev polynomial values $T_{i}\left(t_{j}\right)$ in the grid $t$, where $i=0, \ldots, l-1, j=1, \ldots, k ; F$ is the diagonal matrix with diagonal elements $(l-i) / l$, $i=0, \ldots, l-1$, to derive the arithmetic mean of the sums of orthogonal series.

The calculation of the expansion coefficients is carried out in two stages. First, all columns of the matrix $A$ are transformed into the expansion coefficients that corresponds to the expansion in the variable $y$ :

$$
C=\frac{2}{k} F T_{t} L_{y t} A
$$

Then, the matrix of the expansion coefficients is transposed and the expansion is repeated that corresponds to the expansion in the variable $x$ :

$$
D=\frac{2}{k} F T_{t} L_{x t} C^{\top}
$$

where $C$ and $D$ are the matrices of the expansion coefficients, the intermediate and the resulting ones.

Reconstruction of the approximated function is carried out by a simple summation of orthogonal series:

$$
Z=T_{x}^{\mathrm{\top}} D^{\mathrm{\top}} T_{y}
$$

Let $E$ be the differentiation operator in the space of expansion coefficients. Then the coefficient matrices corresponding to the partial derivatives $p, r, q, t$, and $s(2)$ are as follows:

$$
P=E D ; R=E^{2} D ; Q=D E^{\top} ; T=D\left(E^{\top}\right)^{2} ; S=P E^{\top} .
$$

These formulas exist only if the matrices of expansion coefficients are square.

\section{Data}

To test the method and algorithm proposed, a portion of the Northern Andes measuring $4^{\circ} \times 4^{\circ}$, located between $2^{\circ} \mathrm{S}$ and $2^{\circ} \mathrm{N}$, and $78^{\circ} 30^{\prime} \mathrm{W}$ and $74^{\circ} 30^{\prime} \mathrm{W}$, was selected. The area 
covers regions of Ecuador, Colombia, and Peru including the parts of the Coastal plain, the Andean Range, and the Upper Amazon basin (Fig. 1a). A DEM of the study area was extracted from the global DEM GTOPO30 [34]. The DEM contains 230,880 points (the matrix 480×481); the grid spacing is $30^{\prime \prime}$ (Fig. 1a).

This area and GTOPO30 were selected because this DEM incorporates a high-frequency noise caused by interpolation errors and inaccurate merging of topographic charts having different accuracy. Spatial distribution of the noise in GTOPO30 is uneven and depends on the accuracy of cartographic sources. In particular, the potent noise is typical for forested regions of South America because reasonably detailed and accurate topographic data were unavailable for such areas. Thus, interpolation of sparse contours has been used to compile these portions of GTOPO30. Although DEM noise has no obstacle to produce realistic maps of elevation, it leads to derivation of noisy and unreadable maps of local morphometric variables (computation of the first and the second partial derivatives of elevation dramatically increases the noise [35]). The study area, consisting of two main zones - high mountains and forested foothills - which have different signal-to-noise ratio, is ideally suited to validate the method and algorithm proposed as a tool for DEM analytical treatment. Earlier, this DEM was used to evaluate 2D singular spectrum analysis as a tool to filter DTMs [14].

\section{Data Processing}

Various numbers of expansion coefficients were evaluated to reconstruct DEMs. Finally, the most expressive variants were selected to illustrate capabilities of the method and algorithm to generalize and denoise DEMs as well as to calculate partial derivatives for further computation of local morphometric attributes. In particular, DEMs were reconstructed with 480, 240, 120, 60, and 30 expansion coefficients (Figs. 1a-1e). The first and the second partial derivatives were analytically calculated from the reconstructed DEMs. Digital models of 14 local morphometric attributes were then computed using the derivatives. The models of slope gradient, slope aspect, minimal curvature, maximal curvature, mean curvature, the Gaussian curvature, unsphericity curvature, horizontal curvature, vertical curvature, difference curvature, accumulation curvature, ring curvature, vertical excess curvature, and horizontal excess curvature were derived. To illustrate efficiency of the method and algorithm, $k_{h}$ maps are presented (Figs. 1f $-1 \mathrm{j}$ ).

To estimate the approxamation accuracy, deviations of the reconstructed DEMs were also calculated from the initial DEM (Fig. 2).

Wide dynamic ranges usually characterize topographic variables. To avoid loss of information on spatial distribution of values of morphometric attributes in mapping, it makes sense to apply a logarithmic transform using the following expression [15]:

$$
\Theta^{\prime}=\operatorname{sign}(\Theta) \ln \left(1+10^{n}|\Theta|\right)
$$

where $\Theta$ is the value of a morphometric variable; and $n=0$ for elevation and nonlocal variables and $n=2, \ldots, 18$ for local variables. For the DEM analytical treatment, selection of the $n$ value depends on the size of a study area ( $n=8$ was used for $k_{h}$ mapping). Such a form of logarithmic transformation considers that dynamic ranges of some topographic attributes include both positive and negative values. All DEMs and $k_{h}$ digital models derived were logarithmically transformed to prepare readable maps of elevation and $k_{h}$ (Fig. 1).

The DTMs produced had a grid size of $30^{\prime \prime}$. The plate carrée projection was used for mapping. Data processing and mapping were done by software Matlab R2008b. 

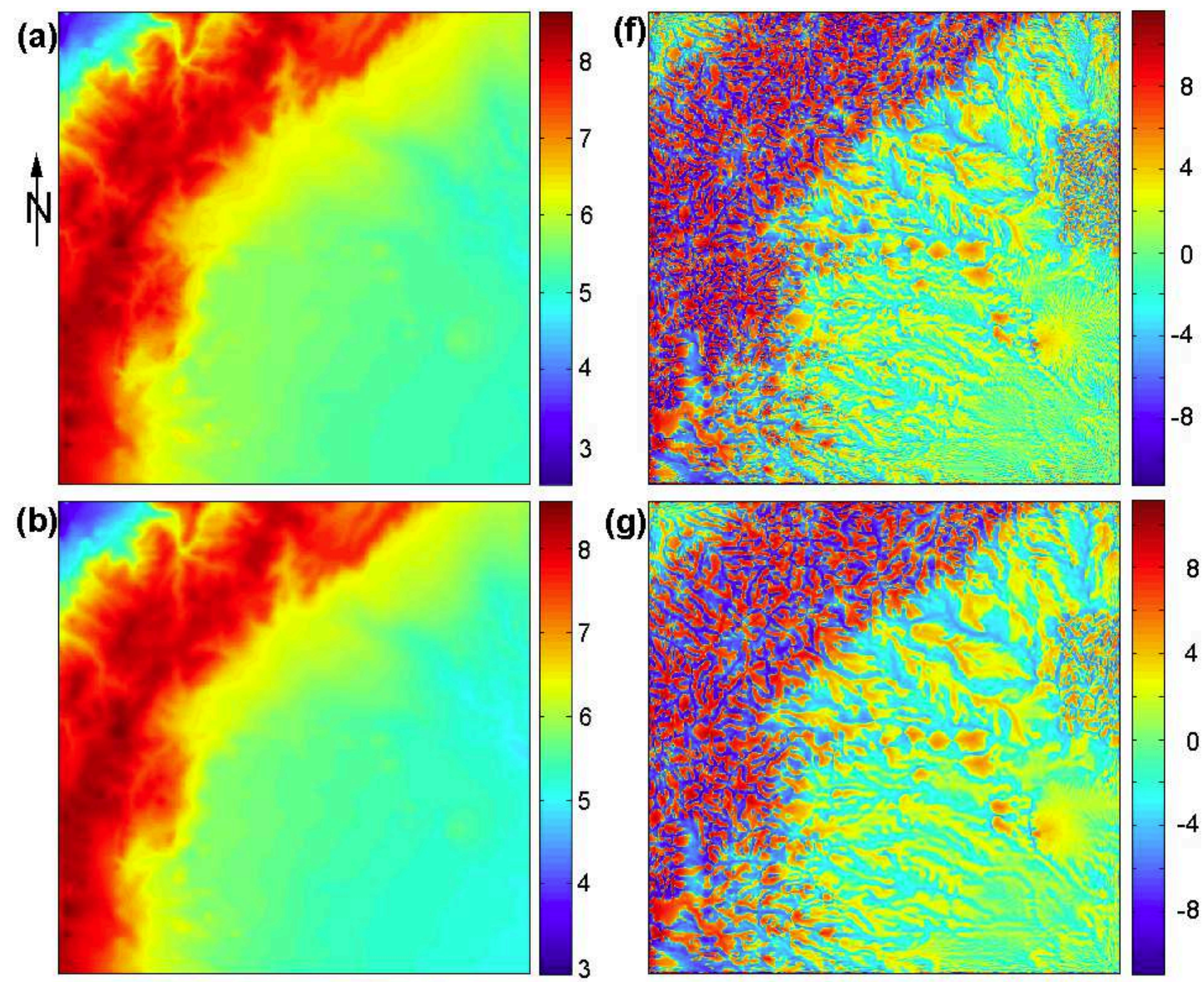

(c)
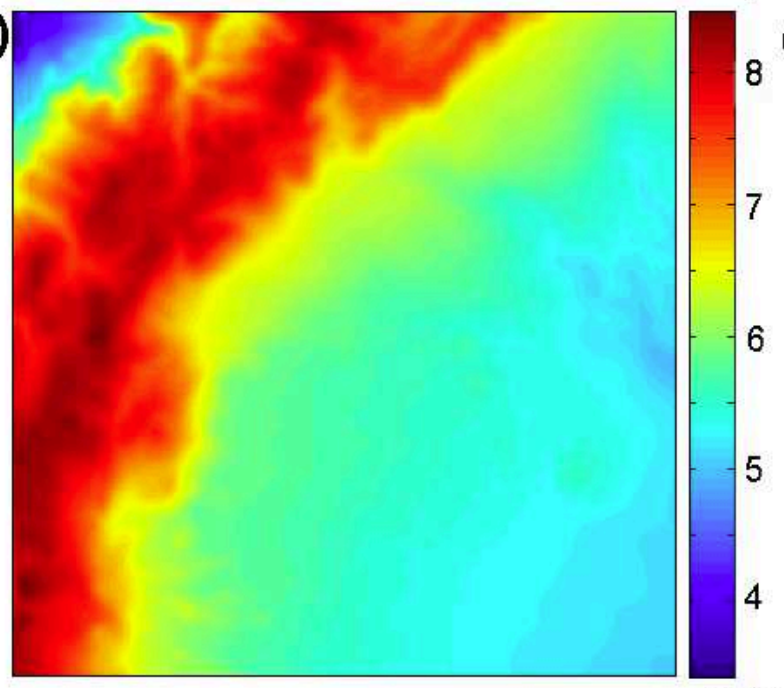

(h)

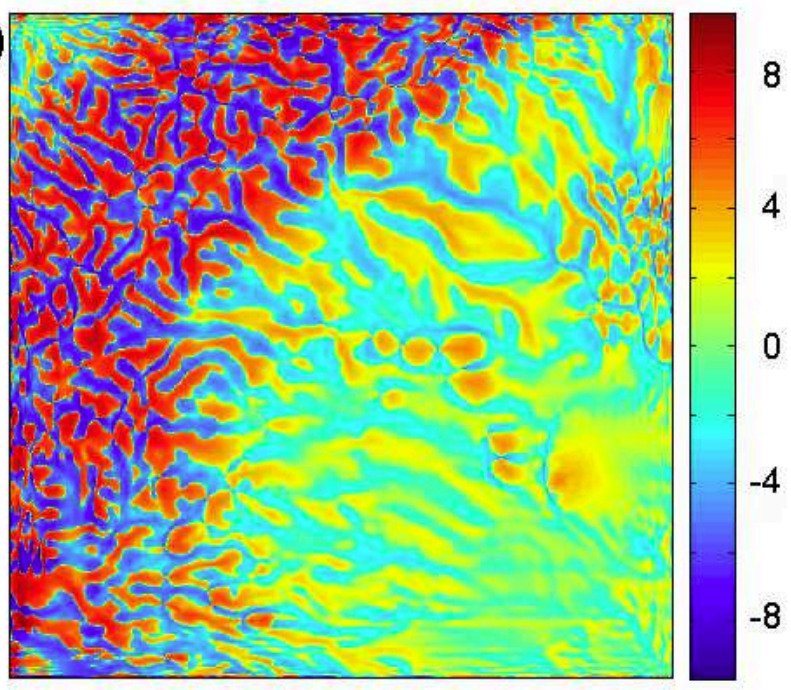

$100 \mathrm{~km}$

Figure 1 The Northern Andes: elevation maps reconstructed with 480 (a), 240 (b), 120 (c), 60 (d), and 30 (e) expansion coefficients; and $k_{h}$ maps derived from reconstructed DEMs with 480 (f), 240 (g), $120(\mathrm{~h}), 60(\mathrm{i})$, and $30(\mathrm{j})$ expansion coefficients. The legends are in logarithmic scale (to be continued) 

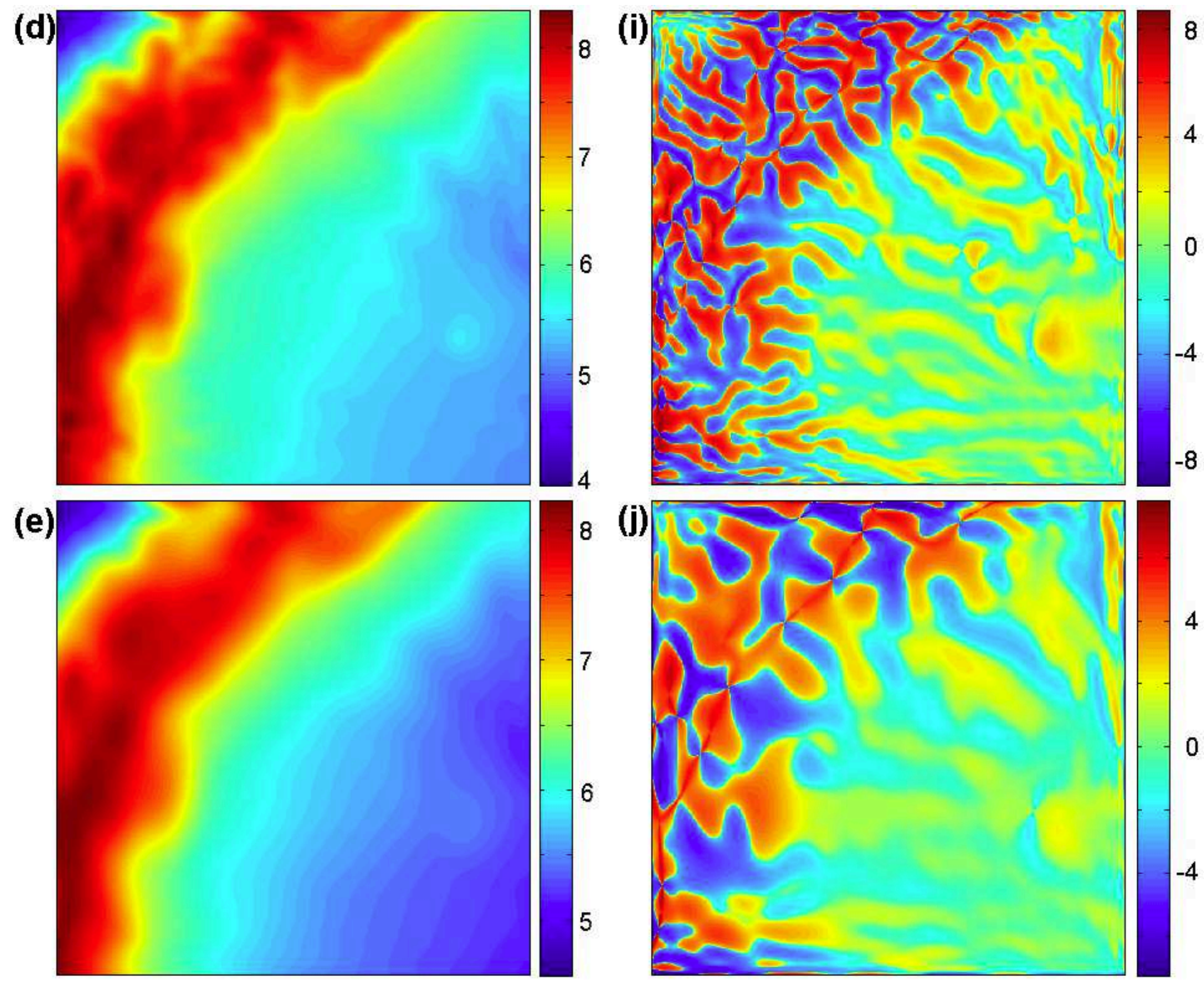

Figure 1 (continued)

\section{Results and Discussion}

\subsection{Visual analysis of maps obtained}

A set of elevation maps reconstructed with 480, 240, 120, 60, and 30 expansion coefficients (Figs. 1a-1e) demonstrates a process of DEM generalization, from its minimal level (Fig. 1a) to the maximal one (Fig. 1e). Similarly, a set of $k_{h}$ maps calculated from DEMs reconstructed with 480, 240, 120, 60, and 30 expansion coefficients (Figs. 1f-1j) shows $k_{h}$ generalization, from its minimal level (Fig. 1f) to the maximal one (Fig. 1j).

The visual comparison of elevation maps reconstructed with 480, 240, and 120 expansion coefficients (Figs. 1a-1c) allows one to see nothing but marginal changes in image patterns. A cursory examination may lead to an underestimation of results of the DEM generalization. The $k_{h}$ maps give better insight into the results. A comparison between $k_{h}$ maps derived from different DEMs (Figs. 1f-1j) shows a pronounced effect of the map generalization. The less number of the expansion coefficients used to reconstruct a DEM, the more smooth and simplified image patterns obtained. One can see the so-called flow structures formed by convergence and divergence areas (negative and positive $k_{h}$ values, respectively). 
(a)

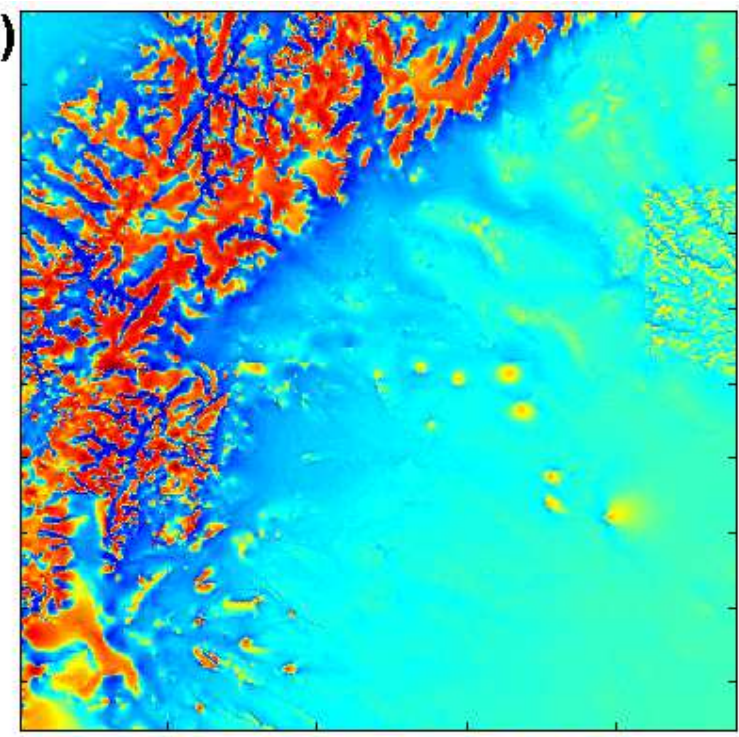

(c)

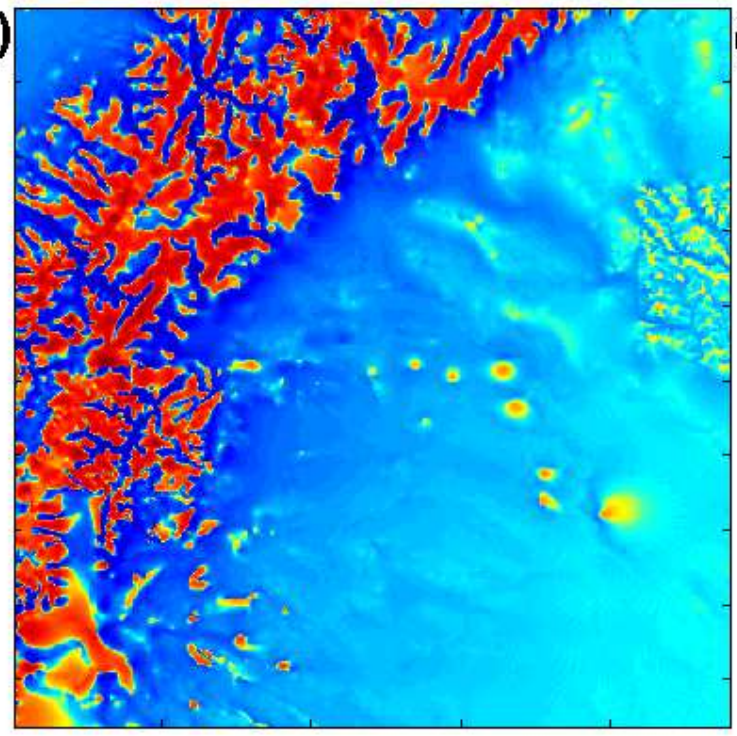

(b)

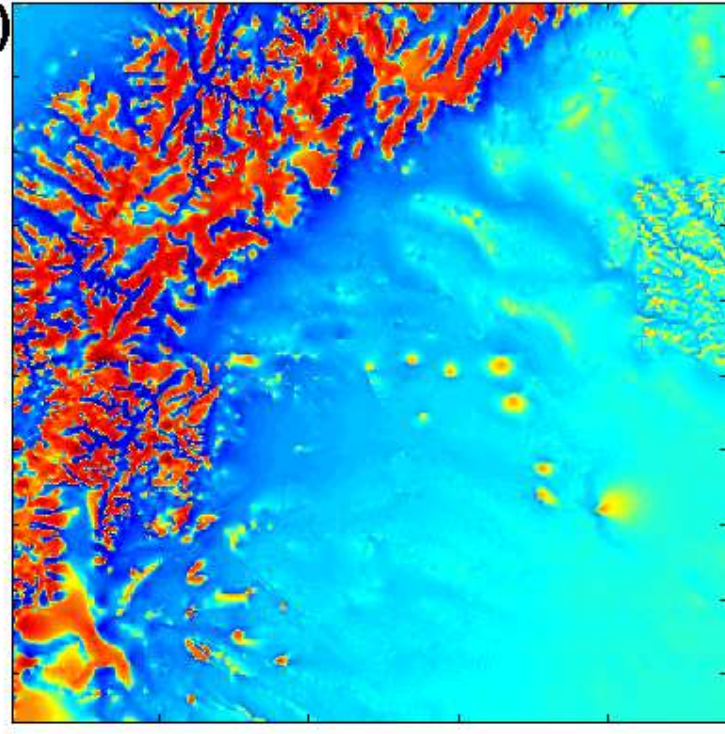

(d)

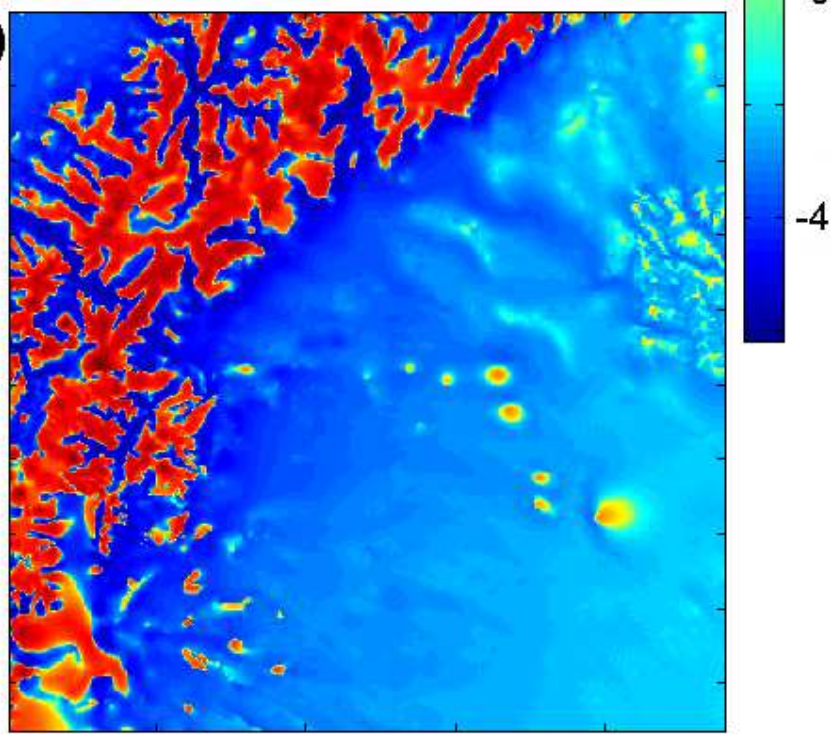

Figure 2 Deviations of the reconstructed DEMs from the initial DEM. Reconstructions were done with 480 (a), 240 (b), 120 (c), and 60 (d) expansion coefficients. The legend is in logarithmic scale

The DEMs reconstructed with 60 and 30 expansion coefficients (Figs. 1d and 1e) are marked by the highest level of generalization. These elevation maps represent a generalized morphostructure of the continental scale, the Andean Range with foothills.

The decrease of the number of the expansion coefficients in DEM reconstruction acts as a high-frequency filtering. Indeed, manifestation of high-frequency noise can be found on the $k_{h}$ map derived from the DEM reconstructed with 480 expansion coefficients (see the bottom right corner in Fig. 1f). This noise is typical for the Andean foothills covered by dense rain forests. However, there are no traces of this noise on $k_{h}$ maps derived from DEMs reconstructed with less number of expansion coefficients (Figs. $1 \mathrm{~g}-1 \mathrm{j}$ ). One can also observe the removal of an artifact of other sort: a rectangular feature along the northeastern border of several $k_{h}$ maps (Figs. 1f-1h). This is a trace of the inaccurate merging of adjacent topographic charts marked by different accuracy during the compilation of GTOPO30. All traces of the artifact disappear on $k_{h}$ maps calculated from DEMs reconstructed with 60 and 30 expansion coefficients (Figs. 1i and $1 \mathrm{j}$ ). 
It is well known that orthogonal polynomial approximation produces boundary effects which cannot be completely eliminated. In the present case, they appear as linear artifacts at the boundaries of $k_{h}$ maps (Figs. 1f-1h). A problem with map boundaries also arises using the finite difference algorithms: it is impossible to estimate partial derivatives for boundary columns and rows of a DEM, because they are estimated for the center point of the moving window.

Note that the above mentioned noise and artifacts are weakly expressed: they are not visible on the elevation maps (Figs. 1a-1e) and become clearly visible only on $k_{h}$ maps (Figs. 1f-1j) after the calculation of partial derivatives of elevation which reinforce their expression.

\subsection{Accuracy of DEM approximation and morphometric calculation}

In digital terrain modeling, it is undesirable to use statistical metrics describing the accuracy of a DTM by a single value. Indeed, the values of elevation and other morphometric variables can be unevenly distributed in space that leads to the spatial variability of the DTM accuracy. To estimate the DTM accuracy, it is appropriate to produce the maps of statistical metrics characterizing DTM accuracy or errors at each point of a DTM (e.g., Fig. 2).

The analysis of Fig. 2 shows that maximum deviations of reconstructed DEMs predictably occur in areas of the contrast mountainous topography with dramatic elevation changes. The use of the global approximation with the Fejér summation leads to a strong smoothing of the elevation function within such areas. However, it is necessary to remember that the goal is twofold: on the one hand, it is required to hold a sufficient approximation accuracy of elevation values using large sets of expansion coefficients in DEM reconstruction and, on the other hand, it is required to suppress high-frequency noise during DEM reconstruction. Since these goals to some extent contradict one another, the key criterion is a sufficient plausibility of reconstructed elevation maps with a simultaneous satisfactory noise reduction.

It is clear that the smaller the number of expansion coefficients used to reconstruct DEMs, the greater the deviation of a DEM reconstructed from the initial DEM (Figs. 2b-2d). We generalize the topographic surface reducing the number of expansion coefficients (Figs. 1c-1e), so the approximation accuracy problem becomes irrelevant.

The local morphometric variables are the functions of measured variables (elevations). Thus, to estimate the accuracy of calculation of local morphometric attributes, one can use the criterion of root mean square error (RMSE) of a function of measured variables. The RMSE equation for $k_{h}$ is as follows $[4,36]$ :

$$
\begin{aligned}
m_{k_{h}}=\frac{1}{p^{2}+q^{2}} & \left(\frac { 1 } { 1 + p ^ { 2 } + q ^ { 2 } } \left\{m_{p}^{2}\left[p\left(q^{2} r-2 p q s+p^{2} t\right)\left(\frac{2}{p^{2}+q^{2}}+\frac{1}{1+p^{2}+q^{2}}\right)+2(q s-p t)\right]^{2}\right.\right. \\
+ & m_{q}^{2}\left[q\left(q^{2} r-2 p q s+p^{2} t\right)\left(\frac{2}{p^{2}+q^{2}}+\frac{1}{1+p^{2}+q^{2}}\right)+2(p s-q r)\right]^{2} \\
& \left.\left.+m_{r}^{2} q^{4}+4 m_{s}^{2} p^{2} q^{2}+m_{t}^{2} p^{4}\right\}\right)^{1 / 2}
\end{aligned}
$$

where $m_{p}, m_{q}, m_{r}, m_{s}$, and $m_{t}$ are the RMSEs of $p, q, r, s$, and $t(2)$, respectively. The RMSE equations of other local morphometric variables can be found elsewhere [4].

Equation (6) can be applied to produce a digital model and map of $m_{k_{h}}$. Earlier, analysis of $m_{k_{h}}$ maps allowed to determine some regularity for spatial distribution of $m_{k_{h}}$ values. Generally, the flatter topography, the higher $m_{k_{h}}$ value $[18,36]$. The $m_{k_{h}}$ values can be in excess of maximum absolute values of $k_{h}$ within flat areas. However, this does not mean that there are errors in DTMs within such areas: $m_{k_{h}}$ is a statistical property of a function $k_{h}$; that is, $m_{k_{h}}$ values represent a possibility for errors. 
Table 1 The average processing time to reconstruct a DEM and to derive a morphometric variable (seconds) depending on the number of expansion coefficients used

\begin{tabular}{ccc}
\hline Number of coefficients & DEM reconstruction & DEM reconstruction $+k_{h}$ derivation \\
\hline 30 & 0.05 & 0.06 \\
60 & 0.06 & 0.07 \\
120 & 0.07 & 0.08 \\
240 & 0.08 & 0.09 \\
480 & 1.40 & 1.50 \\
960 & 3.10 & 3.20 \\
1200 & 4.40 & 4.50 \\
2400 & 13.60 & 13.70 \\
3000 & 20.50 & 20.60 \\
\hline
\end{tabular}

\subsection{Efficiency of the method and algorithm}

To estimate the efficiency of the method and algorithm developed, an office computer with modest facilities (Intel Celeron, CPU G460, $1.80 \mathrm{GHz}, 1.95 \mathrm{~GB}$ RAM) was used. Test results are presented in Table 1. It is clearly seen that most of the time is spent on the DEM reconstruction; it nonlinearly depends on the number of expansion coefficients used. In this regard, the time for calculation of partial derivatives and local morphometric variables is absolutely negligible.

Earlier, the initial DEM was used to evaluate 2D singular spectrum analysis as a tool to generalize, smooth, and denoise DTMs; the software 2D-SSA was utilized [14]. The processing time and DEM treatment results were, in general, comparable to those presented in this paper. However, 2D singular spectrum analysis does not allow one to calculate analytically partial derivatives and morphometric variables.

The processing time to derive a local morphometric variable by a finite difference algorithm from the initial DEM was estimated as about $0.5 \mathrm{~s}$ (the software LandLord [4] was applied). So, the efficiency of the developed method and algorithm is comparable with that of existing finite difference ones.

The advantage of the developed method and algorithm is the possibility to apply several procedures of DTM treatment — DEM approximation, generalization, smoothing, as well as calculation of partial derivatives and morphometric variables - within the framework of a single approach. These procedures are usually implemented in individual alogorithms, utilities, or even programs. Such integration allows one to save time for DTM processing.

\section{Concluding Remarks}

The study demonstrated a good performance of the developed method and algorithm. They can be utilized as a universal tool for analytical treatment of regularly spaced DEMs including DEM global approximation, denoising, and generalization as well as derivation of local morphometric variables from DEMs using analytical calculation of partial derivatives. Further development of the method will include: (a) incorporation of other high-order orthogonal polynomials (e. g., the Fourier and Legendre ones); (b) their comparative analysis in terms of efficiency and accuracy of DEM treatment; (c) evaluation of calculation accuracy using appropriate statistical metrics (e. g., RMSE of a function of the measured values); and (d) comparison of the developed method and algorithm with traditional techniques applied in digital terrain modeling. 


\section{References}

[1] Wilson, J.P., and J.C. Gallant, eds. 2000. Terrain analysis: Principles and applications. New York, NY: Wiley. 479 p.

[2] Li, Z., Q. Zhu, and C. Gold. 2005. Digital terrain modeling: Principles and methodology. New York, NY: CRC Press. 323 p.

[3] Hengl, T., and H. I. Reuter, eds. 2009. Geomorphometry: Concepts, software, applications. Amsterdam: Elsevier. 796 p.

[4] Florinsky, I. V. 2012. Digital terrain analysis in soil science and geology. Amsterdam: Elsevier / Academic Press. 379 p.

[5] Schut, G.H. 1976. Review of interpolation methods for digital terrain models. Can. Surv. 30(5):389-412.

[6] Watson, D. 1992. Contouring: A guide to the analysis and display of spatial data. Oxford: Pergamon. 340 p.

[7] Mitas, L., and H. Mitasova. 1999. Spatial interpolation. Geographical information systems: Principles, techniques, management and applications. 2nd ed. Hoboken: Wiley. 481-492.

[8] Papo, H. B., and E. Gelbman. 1984. Digital terrain models for slopes and curvatures. Photogramm Eng. Remote Sens. 50(6):695-701.

[9] Harrison, J. M., and C.-P. Lo. 1996. PC-based two-dimensional discrete Fourier transform programs for terrain analysis. Comp. Geosci. 22(4):419-424.

[10] Arrell, K., S. Wise, J. Wood, and D. Donoghue. 2008. Spectral filtering as a method of visualising and removing striped artefacts in digital elevation data. Earth Surf. Proc. Landforms 33(6):943961.

[11] Bergbauer, S., T. Mukerji, and P. Hennings. 2003. Improving curvature analyses of deformed horizons using scale-dependent filtering techniques. Amer. Assoc. Petrol Geol. Bull. 87(8):12551272 .

[12] Bjørke, J.T., and S. Nilsen. 2003. Wavelets applied to simplification of digital terrain models. Int. J. Geogr. Inf. Sci. 17(7):601-621.

[13] Wu, F. 2003. Scale-dependent representations of relief based on wavelet analysis. Geo-Spat. Inf. Sci. 6(1):66-69.

[14] Golyandina, N. E., K. D. Usevich, and I. V. Florinsky. 2007. Filtering of digital terrain models by two-dimensional singular spectrum analysis. Int. J. Ecol. Devel. 8(F07):81-94.

[15] Shary, P. A., L. S. Sharaya, and A. V. Mitusov. 2002. Fundamental quantitative methods of land surface analysis. Geoderma 107(1-2):1-32. doi:10.1016/S0016-7061(01)00136-7.

[16] Evans, I. S. 1979. Statistical characterization of altitude matrices by computer. An integrated system of terrain analysis and slope mapping. Final Report on Grant DA-ERO-591-73-G0040. Durham: Department of Geography, University of Durham. 192 p.

[17] Zevenbergen, L. W., and C. R. Thorne. 1987. Quantitative analysis of land surface topography. Earth Surf. Proc. Landforms 12(1):47-56. doi:10.1002/esp.3290120107.

[18] Florinsky, I. V. 1998. Derivation of topographic variables from a digital elevation model given by a spheroidal trapezoidal grid. Int. J. Geogr. Inf. Sci. 12(8):829-852. doi:10.1080/136588198241527.

[19] Florinsky, I. V. 2009. Computation of the third-order partial derivatives from a digital elevation model. Int. J. Geogr. Inf. Sci. 23(2):213-231. doi:10.1080/13658810802527499.

[20] Heifetz, B. S. 1964. Approximation of the topographic surface by the Chebyshev orthogonal polynomials. Izv. Vuzov. Geod. Aerofoto 2:78-86. (In Russian.)

[21] Van Rossel, J. 1972. Digital hypsographic map compilation. Photogramm Eng. 38(11):1106-1116. 
[22] Yagodina, L. L. 1972. Modelling of the topographic field by the Chebyshev orthogonal polynomials. Vest. Leningrad Univ. Ser. Geogr. 24(4):136-142 (In Russian.)

[23] Segu, W. P. 1985. Terrain approximation by fixed grid polynomial. Photogramm Rec. 11(65):581591. doi:10.1111/j.1477-9730.1985.tb00525.x.

[24] Chorley, R. J., and P. Huggett. 1965. Trend-surface mapping in geographical research. Trans. Inst. Brit. Geogr. 37:47-67. doi:10.2307/621689.

[25] Tobler, W.R. 1969. Geographical filters and their inverses. Geogr. Anal. 1(3):234-253. doi:10.1111/j.1538-4632.1969.tb00621.x.

[26] Dedus, F.F., S. A. Makhortykh, M. N. Ustinin, and A.F. Dedus. 1999. Generalized spectral analytical method for data set processing. Problems of image analysis and pattern recognition. Moscow: Mashinostroenie. 356 p. (In Russian, with English abstract.)

[27] Dedus, F. F., L. I. Kulikova, A. N. Pankratov, and R. K. Tetuev. 2004. Classical orthogonal bases in problems of analytical description and processing of information signals. Moscow: Moscow State Univ. 168 p. (In Russian.)

[28] Tetuev, R. K., and F. F. Dedus. 2007. Classical orthogonal polynomials. Application to data processing problems. Pushchino: Institute of Mathematical Problems of Biology, Russian Academy of Sciences. 60 p. (In Russian.)

[29] Press, W. H., S. A. Teukolsky, W. T. Vetterling, and B. P. Flannery. 1992. Numerical recipes in C. The art of scientific computing. 2nd ed. Cambridge: Cambridge Univ. Press. 994 p.

[30] Pankratov, A. N. 2004. On the implementation of algebraic operations on orthogonal function series. Comp. Math. Math. Phys. 44(12):2017-2023.

[31] Jerri, A. J. 1998. The Gibbs phenomenon in Fourier analysis, splines and wavelet approximations. Boston: Kluwer. 336 p. doi:10.1007/978-1-4757-2847-7.

[32] Courant, R., and D. Hilbert. 1989. Methods of mathematical physics. Vol. 1. New York, NY: Wiley. 560 p. doi:10.1002/9783527617210.

[33] Pankratov, A. N., and L. I. Kulikova. 2006. On the derivatives calculation under uniform and mean square approximation of signals. Computer Applications in Scientific Researches Proceedings, IVTN-2006 Session. Moscow. 56.

[34] U.S. Geological Survey. 1996. GTOPO30, a 30-arc seconds global digital elevation model. Available at: https://1ta.cr.usgs.gov/GTOPO30 (accessed August 12, 2015).

[35] Florinsky, I. V. 2002. Errors of signal processing in digital terrain modelling. Int. J. Geogr. Inf. Sci. 16(5):475-475. doi:10.1080/13658810210129139.

[36] Florinsky, I. V. 1998. Accuracy of local topographic variables derived from digital elevation models Int. J. Geogr. Inf. Sci. 12(1):47-61. doi:10.1080/136588198242003. 